\title{
Sirtuins and Chronic Obstructive Pulmonary Disease
}

\author{
C. Carollo*, C. Urso, R. Lo Presti, G. Caimi \\ Biomedical Department of Internal and Specialistic Medicine, University of Palermo, Palermo, Italy \\ Email: *caterina.carollo@unipa.it
}

How to cite this paper: Carollo, C., Urso, C., Lo Presti, R. and Caimi, G. (2018) Sirtuins and Chronic Obstructive Pulmonary Disease. Food and Nutrition Sciences, 9, 1254-1260.

https://doi.org/10.4236/fns.2018.910089

Received: September 12, 2018

Accepted: October 26, 2018

Published: October 30, 2018

Copyright (๑) 2018 by authors and Scientific Research Publishing Inc. This work is licensed under the Creative Commons Attribution International License (CC BY 4.0).

http://creativecommons.org/licenses/by/4.0/

(c) (i) Open Access

\begin{abstract}
Chronic obstructive pulmonary disease (COPD) is one of the world leading causes of death. It has been recently related to aging and inflammation, as well as sirtuins are actually recognized to be involved in both these phenomena. Sirtuins are a family of highly conserved protein deacetylases and they influence the factors that worsen physiological aging such as glucose metabolism, DNA stability and cancer, neurodegenerative processes, etc. Among the seven sirtuins, SIRT1 and SIRT6 have been deeply investigated in COPD. Actually researchers hypothesize that SIRT 1 activators could play a fundamental role in counteract COPD. Natural compounds as Resveratrol are actually retained SIRT1 activators and could be indicated as powerful weapons against this complex disease.
\end{abstract}

\section{Keywords}

Sirtuins, Chronic Obstructive Pulmonary Disease, Resveratrol, Wine

\section{Introduction}

Chronic obstructive pulmonary disease (COPD) is one of the ten leading causes of total years life lost in 2016 [1].

It also requires the greatest amount of health care utilization [2] also because patients frequently undergo exacerbations [3] and almost all of them have comorbidities [4], thus negatively influencing their work ability and global social costs [5].

In the last decade, a growing body of evidences lead researchers to consider COPD as an age-related disease: In fact an increased prevalence in elder population along with the presence in COPD of morpho-structural alterations similar to what observed in old lung, has been widely described [6].

Many aging features such as telomere shortening, cellular senescence, impaired 
autophagy, mitochondrial dysfunction, stem cell lost, genetic and epigenetic modifications, and a low-grade chronic inflammation (the so-called inflammaging)

[7] are evident in COPD and some of them are strictly correlated with smoke habit and number of pack-years [8].

The most of the above mentioned processes are strictly interrelated and in different manners they are involved in pathophysiology of COPD [9].

Along with these observations, antiaging molecules have been widely recognised to be reduced in COPD. As a consequence, cell agents that regulate these phenomena have been deeply investigated. Among them, Sirtuins are worthy of consideration.

\section{Sirtuins}

Sirtuins are a family of highly conserved protein deacetylases that depend on nicotinamide adenine dinucleotide for their activity. They catalyze the removal of acetyl groups from lysine residua. They may promote different post translational modifications in many different proteins so they are actually known as deacylases [10].

Up to now, seven sirtuins have been recognized, listed from 1 to 7 . Each of them shows a catalytic domain (present in all sirtuins) whilst different $\mathrm{N}$ - and C-ends give selective biological features to every sirtuin.

In a previous paper [11] we examined the role of sirtuins in influencing the factors that worsen physiological aging such as glucose metabolism, DNA stability and cancer, neurodegenerative processes, etc.

Now our aim was to clarify the role of sirtuins as pathophysiological agents and therapeutical targets in COPD.

\section{Sirtuins and COPD}

Among the different Sirtuins, SIRT1 (the most deeply investigated sirtuin in mammals) plays a fundamental role in influencing oxidative cell damage and, as a consequence, health and life duration [12]. In fact, it is involved in glucose metabolism, DNA reparation, etc. by modulating oxidative stress response, endothelial dysfunction and inflammation.

Many years ago, Sirt1 levels were found to be reduced in lungs of smokers and COPD patients [13] and this finding was inversely related to increased amounts of oxidative and nitrosative markers. A few years later, another group of authors demonstrated that while SIRT1 decreases, prosenescent factors, such as ciclin-dependent kinase inhibitor 2 and caveolin 1, increase in COPD severity-dependent manner [14] whilst SIRT1 overexpression modulates this phaenomenum [15] and protects against emphysema by counteracting the premature senescence in mice.

In this animal model, these researchers demonstrated the leading role of SIRT1 in attenuating aging-associated manifestations of COPD: interestingly, SIRT1 expression and functional acivity decrease along with age progression. 
Last year, for the first time, serum SIRT1 levels were found to be decreased in COPD patients and this reduction was positively correlated with airway obstruction entity, severity of lung emphysema and frequency of exacerbations [16].

More recently, SIRT1 has been demonstrated to protect lungs in the early and late stages of pulmonary fibrosis in patients with systemic sclerosis by reducing proinflammatory and profibrotic processes [17]. Also SIRT7 has been involved in pulmonary fibrosis: in fact its levels are reduced in rat model of this disease and are associated with a profibrotic fenotype of lung fibroblasts [18].

Also cardiovascular function, which is impaired in COPD patients, is positively influenced by SIRT1 which is able to regulate vascular senescence and atherosclerosis development [19].

In addition to SIRT1, SIRT6 have also been shown to improve COPD. SIRT6 is associated with redox state and inhibits cellular senescence and fibrosis [20] [21]. It is also involved in genomic stability, DNA damage response, inflammation, cell senescence and aging [22] so it could be another putative therapeutical target for natural and synthetic compounds.

For all the above described pathophysiological implications, SIRT1 and SIRT6 have been recently identified as the leading targets for a new therapeutical approach in the treatment of COPD [23].

\section{Sirtuin Modulation}

Different dietary polyphenols such as quercetin, curcumin, catechins and resveratrol have been demonstrated to be able in activating (both directly and indirectly) SIRT1 in addiction to their well known antioxidant and antiinflammatory properties.

Among these natural compounds, Resveratrol plays a leading role. In fact it lowers the Km of SIRT1 for NAD+ [24].

In rat models of COPD, treatment with Resveratrol leads to a significant $(\mathrm{p}<$ 0.05 ) reduction of inflammation markers levels (such as IL-6 and IL-8) and reconstruction of small airways [25].

As you know, Resveratrol is a fundamental part of grape. Previously [26] [27] [28] we underlined the beneficial effects of moderate wine consumption in different clinical conditions. In general population [29] wine intake is positively associated with the Forced Expiratory Volume in 1 second (FEV1) [30]. Among wines, the white ones exhibit a positive association with higher levels of FEV1 and FEV1/FVC (Forced Vital Capacity) ratio [30].

These beneficial effects could be enhanced by quercetin, a powerful catechin which was demonstrated to prevent progression of emphysema in mice by reducing oxidative stress, inflammation and lung expression of matrix metalloproteinase activity via an increase of SIRT1 levels [31].

Many Authors investigated about synthetic pharmacological SIRT1 activators and their effects in lung diseases. Interesting results show positive influence of these compounds on septic-associated lung injury [32] [33] emphysema [34] and 
asthma [35]

\section{Conclusions}

SIRT1 activators are promising therapeutical weapons to counteract COPD, as demonstrated by ITO [36] who created the fascinating word "Geroprotectors" to indicate all these agents that fight aging and aging-related disorders.

Could Resveratrol also be considered a Geroprotector? This topic needs further investigations but is undoubtedly worthy of our future attention.

If we consider that "prevent is better than cure", it is reasonable to imagine that a healthy lifestyle, with salubrious foods and beverages, along with a regular exercise, will be a cheap and smart medical prescription in a general population who is always older.

\section{Conflicts of Interest}

The authors declare no conflicts of interest regarding the publication of this paper.

\section{References}

[1] GBD 2016 Causes of Death Collaborators (2017) Global, Regional and Age-Sex Specific Mortality for 264 Causes of Death, 1980-2016: A Systematic Analysis for the Global Burden of Disease Study 2016. Lancet, 390, 1151-1210.

[2] Ford, E.S. (2015) Hospital Discharges, Readmissions, and ED Visits for COPD or Bronchiectasis among US Adults: Finding from the Nationwide Impatient Sample 2001-2012 and Nationwide Emergency Department Sample 2006-2011. Chest, 147, 989-998. https://doi.org/10.1378/chest.14-2146

[3] Dhamane, A.D., Moretz, C., Zhou, Y., Burslem, K., Saverno, K., Jain, G., Renda, A. and Kaila, S. (2015) COPD Exacerbations Frequency and Its Association with Health Care Resource Utilization and Costs. International Journal of Chronic Obstructive Pulmonary Disease, 10, 2609-2618. https://doi.org/10.2147/COPD.S90148

[4] Schwab, P., Dhamane, A.D., Hopson, S.D., Moretz, C., Annavarapu, S., Burslem, K., Renda, A. and Kaila, S. (2017) Impact of Comorbid Conditions in COPD Patients oh Health Care Resource Utilization and Costs in a Predominantly Medicare Population. International Journal of Chronic Obstructive Pulmonary Disease, 12, 735-744. https://doi.org/10.2147/COPD.S112256

[5] Dhamane, A.D., Witt, E.A. and Su, J. (2016) Associations between COPD Severity and Work Productivity, Health-Related Quality of Life and Health-Care Resource Use: A Cross-Sectional Analysis of National Survey Data. Journal of Occupational and Environmental Medicine, 58, e191-e197. https://doi.org/10.1097/JOM.0000000000000735

[6] Ito, K. and Barnes, P.J. (2009) COPD as a Disease of Accelerated Lung Aging. Chest, 135, 173-180. https://doi.org/10.1378/chest.08-1419

[7] Barnes, P.J. (2017) Senescence in COPD and Its Comorbidities. Annual Review of Physiology, 79, 517-539. https://doi.org/10.1146/annurev-physiol-022516-034314

[8] Rutten, E.P.A., Gopal, P., Wouters E.F.M., Franssen, F.M.E., Hageman, G.J., Vanfleteren, L.E., Spruit, M.A. and Reynaert, N.L. (2016) Various Mechanistic Pathways Representing the Aging Process Are Altered in COPD. Chest, 149, 53-61. 
https://doi.org/10.1378/chest.15-0645

[9] Yao, H. and Rahman, I. (2011) Current Concepts on Oxidative/Carbonyl Stress, Inflammation and Epigenetics in Pathogenesis of Chronic Obstructive Pulmonary Disease. Toxicology and Applied Pharmacology, 254, 72-85. https://doi.org/10.1016/j.taap.2009.10.022

[10] Verdin, E., Hirschey, M.D., Finley, L.W. and Haigis, M.C. (2010) Sirtuin Regulation of Mitochondria: Energy Production, Apoptosis and Signalling. Trends in Biochemical Sciences, 35, 669-675. https://doi.org/10.1016/j.tibs.2010.07.003

[11] Carollo, C., Firenze, A. and Caimi, G. (2016): Sirtuins and Aging: Is There a Role for Resveratrol? International Journal of Advanced Nutritional and Health Science, 4, 203-211.

[12] Salminen, A., Kaarniranta, K. and Kauppinen, A. (2013) Crosstalk between Oxidative Stress and SIRT1: Impact on the Aging Process. International Journal of Molecular Sciences, 14, 3834-3859. https://doi.org/10.3390/ijms14023834

[13] Rajendrasozhan, S., Yang, S.R., Kinnula, V.L. and Rahman, I. (2008) SIRT1, an Antiinflammatory and Antiaging Protein, Is Decreased in Lungs of Patients with Chronic Obstructive Pulmonary Disease. American Journal of Respiratory and Critical Care Medicine, 177, 861-870. https://doi.org/10.1164/rccm.200708-1269OC

[14] Acquaah-Mensah, G.K., Malhotra, D., Vulimiri, M., McDermott, J.E. and Biswal, S. (2012) Suppressed Expression of T-Box Transcription Factors Is Involved in Senescence in Chronic Obstructive Pulmonary Disease. PLOS Computational Biology, 8 , e1002597. https://doi.org/10.1371/journal.pcbi.1002597

[15] Yao, H. and Rahman, I. (2012) Role of Histone Deacetylase 2 in Epigenetics and Cellular Senescence: Implications in Lung Inflammation and COPD. American Journal of Physiology-Lung Cellular and Molecular Physiology, 303, L557-L566. https://doi.org/10.1152/ajplung.00175.2012

[16] Yanagisawa, S., Papaioannou, A.I., Papaporfyriou, A., Baker, J.R., Vuppusetty, C., Loukides, S., Barnes, P.J. and Ito, K. (2017) Decreased Seum Sirtuin 1 in COPD. Chest, 152, 343-352. https://doi.org/10.1016/j.chest.2017.05.004

[17] Chu, H., Jiang, S., Liu, Q., Ma, Y., Zhu, X., Liang, M., Shi, X., Ding, W., Zhou, X., Zou, H., Qian, F., Shaul, P.W, Jin, L. and Wang, J. (2018) Sirtuin 1 Protects against Systemic Sclerosis-Related Pulmonary Fibrosis by Decreasing Proinflammatory and Profibrotic Processes. American Journal of Respiratory Cell and Molecular Biology, 58, 28-39. https://doi.org/10.1165/rcmb.2016-0192OC

[18] Wyman, A.E., Noor, Z., Fishelevich, R., Lockatell, V., Shah, N.G., Todd, N.W. and Atamas, S.P. (2017) Sirtuin 7 Is Decreased in Pulmonary Fibrosis and Regulates the Fibrotic Phenotype of Lung Fibroblasts. American Journal of Physiology-Lung Cellular and Molecular Physiology, 312, L945-L958.

https://doi.org/10.1152/ajplung.00473.2016

[19] Arunachalam, G., Yao, H., Sundar, I.K., Caito, S. and Rahman, I. (2010) SIRT1 Regulates Oxidant- and Cigarette Smoke-Induced eNOS Acetylation in Endothelial Cells: Role of Resveratrol. Biochemical and Biophysical Research Communications, 393, 66-72. https://doi.org/10.1016/j.bbrc.2010.01.080

[20] Ota, H., Eto, M., Kano, M.R., Kahyo, T., Setou, M., Ogawa, S., Iijima, K., Akishita, M. and Ouchi, Y. (2010) Induction of Endothelial Nitric Oxide Synthase, SIRT1, and Catalase by Statins Inhibits Endothelial Senescence through the Akt Pathway. Arteriosclerosis, Thrombosis, and Vascular Biology, 30, 2205-2211. https://doi.org/10.1161/ATVBAHA.110.210500

[21] Corbi, G., Manzo, V., Pelaia, G., Filippelli, A. and Vatrella, A. (2015) Sirtuin 1 and 
Aging Theory for Chronic Obstructive Pulmonary Disease. Analytical Cellular Pathology, 2015, Article ID: 897327.

[22] Rahman, I., Kinnula, V.L., Gorbunova, V. and Yao, H. (2012) SIRT 1 as a Therapeutic Target in Inflammaging of the Pulmonary Disease. Preventive Medicine, 54, s20-s28. https://doi.org/10.1016/j.ypmed.2011.11.014

[23] Chun, P. (2015) Role of Sirtuins in Chronic Obstructive Pulmonary Disease. Archives of Pharmacal Research, 38, 1-10.

[24] Zhang, Z., Lowry, S.F., Guarente, L. and Haimovich, B. (2010) Role of Sirt 1 in the Acute and Restorative Phases Following Induction of Inflammation. The Journal of Biological Chemistry, 285, 41391-41401. https://doi.org/10.1074/jbc.M110.174482

[25] Wang, X.L., Li, T., Li, J.H., Miao, S.Y. and Xiao, X.Z. (2017) The Effects of Resveratrol on Inflammation and Oxidative Stress in a Rat Model of Chronic Obstructive Pulmonary Disease. Molecules, 22, E1529.

[26] Lo Presti, R., Carollo, C. and Caimi, G. (2007) Wine Consumption and Renal Diseases: New Perspectives. Nutrition, 23, 598-602.

https://doi.org/10.1016/j.nut.2007.04.012

[27] Carollo, C., Lo Presti, R. and Caimi, G. (2007) Wine, Diet and Arterial Hypertension. Angiology, 58, 92-96. https://doi.org/10.1177/0003319706295478

[28] Caimi, G., Carollo, C. and Lo Presti, R. (2003) Wine and Endothelial Function. Drugs under Experimental and Clinical Research, 29, 235-242.

[29] Schunemann, H.J., Grant, B.J., Freudenheim, J.L., Muti, P., McKan, S.E., Kudalkar, D., Ram, M., Nochajski, T., Russell, M. and Trevisan, M. (2002) Beverage Specific Alcohol Intake in a Population-Based Study: Evidence for a Positive Association between Pulmonary Function and Wine Intake. BMC Pulmonary Medicine, 2, 3. https://doi.org/10.1186/1471-2466-2-3

[30] Siedlinski, M., Boer, J.M.A., Smit, H.A., Postma, D.S. and Boezen, H.M. (2012) Dietary Factors and Lung Function in the General Population: Wine and Resveratrol Intake. European Respiratory Journal, 39, 385-391. https://doi.org/10.1183/09031936.00184110

[31] Ganesan, S., Faris, A.N., Comstock, A.T., Chatoraj, S.S., Chatoraj, A., Burgess, J.R., Curtis, J.L., Martinez, F.J., Zick, S., Hershenson, M.B. and Sajjan, U.S. (2010) Quercetin Prevents Progression of Disease in Elastase/LPS-Exposed Mice by Negatively Regulating MMP Expression. Respiratory Research, 11, 131. https://doi.org/10.1186/1465-9921-11-131

[32] Zhuo, Y., Zhang, S., Li, C., Yang, L., Gao, H. and Wang, X. (2018) Resolvin D1 Promotes SIRT1 Expression to Counteract the Activation of STAT3 and NF- $\kappa$ B in Mice with Septic-Associated Lung Injury. Inflammation, 41, 1762-1771. https://doi.org/10.1007/s10753-018-0819-2

[33] Cheng, C.Y., Gutierrez, N.M., Marzuki, M.B., Lu, X., Foreman, T.W., Pqaleja, B., Lee, B., Balachander, A., Chen, J., Tsenova, L., Kurepina, N., Teng, K.W.W., West, K., Mehra, S., Zolezzi, F., Poidinger, M., Kreiswirth, B., Kaushal, D., Kornfeld, H., Newell, E.W. and Singhal, A. (2017) Host Sirtuin 1 Regulates Mycobacterial Immunopathogenesis and Represents a Therapeutic Target against Tuberculosis. Science Immunology, 2, pii: eaaj1789. https://doi.org/10.1126/sciimmunol.aaj1789

[34] Gu, C., Li, Y., Xu, W.L., Yan, J.P., Xia, Y.J., Ma, Y.Y., Chen, C., Wang, H.J. and Tao, H.Q. (2015) Sirtuin 1 Activator SRT1720 Protects against Lung Injury via Reduction of Type II Alveolar Epithelial Cells Apoptosis in Emphysema. COPD, 12, 444-452. https://doi.org/10.3109/15412555.2014.974740 
[35] Ichikawa, T., Hayashi, R., Suzuki, K., Imanishi, S., Kambara, K., Okazawa, S., Inomata, M., Yamada, T., Yamazaki, Y., Koshimizu, Y., Miwa, T., Matsui, S., Usui, I., Urakaze, M., Matsuya, Y., Sasahara, M. and Tobe, K. (2013) Sirtuin 1 Activator SRT1720 Suppresses Inflammation in an Ovalbumin-Induced Mouse Model of Asthma. Respirology, 18, 332-339. https://doi.org/10.1111/j.1440-1843.2012.02284.x

[36] Ito, K. and Mercado, N. (2014) STOP Accelerating Lung Aging for the Treatment of COPD. Experimental Gerontology, 59, 21-27.

https://doi.org/10.1016/j.exger.2014.03.014 